\title{
The LUCID detector ATLAS luminosity monitor and its electronic system
}

\author{
F. Lasagni Manghi ${ }^{\mathrm{a}, \mathrm{b}}$, ATLAS LUCID collaboration \\ ${ }^{a}$ University of Bologna, Italy \\ ${ }^{b}$ INFN Bologna, Italy
}

\begin{abstract}
In 2015 LHC is starting a new run, at higher center of mass energy (13 TeV) and with 25 ns bunch-spacing. The ATLAS luminosity monitor LUCID has been completely rebuilt, both the detector and the electronics, in order to cope with the new running conditions. The new detector electronics features a new read-out board (LUCROD) for signal acquisition and digitization, PMT-charge integration and single-side luminosity measurements, and a revisited LUMAT board for combination of signals from the two detectors. This note describes the new board design, the firmware and software developments, the implementation of luminosity algorithms, the optical communication between boards and the integration into the ATLAS TDAQ system.
\end{abstract}

\section{LUCID}

The measurement of luminosity is essential in any highenergy physics experiment for cross-section measurements. Since the start of the Large Hadron Collider, LUCID has been the only dedicated luminosity monitor in the ATLAS experiment [1][2]. LUCID is and was made of two modules placed around the beam-pipe on both forward ends of ATLAS. Each module of LUCID is composed of 16 photomultipliers (PMTs)close to the beam-pipe and 4 quartz fibre bundles read by PMTs in a shielded location. The PMTs detect charged particles crossing their quartz window, where Cherenkov light is produced. Light is produced in the fibres as well and carried to PMTs sitting behind shielding a few meters away [3]. A new calibration system for the PMTs allows frequent on-line calibration to compensate for possible yield variations due to ageing.

\section{PMT Calibration}

LUCID II uses R760 Hamamatsu PMTs, a smaller version of the previously used R762 model. The new PMTs have been tested for gamma and neutron radiation hardness, ensuring stable results in ATLAS until 2018. PMT calibrations have been performed with the goal of finding a good working point at low gain, to reduce the current produced in the PMT, thus reducing their ageing. The calibrations were accomplished using Bi-207 sources, that produce monochromatic electrons from interntal conversion, which produce Cherenkov light close to that expected for particles in ATLAS. Shown in Fig. 1 is the amplitude spectrum of the Bi-207 source as recorded by the new PMTs. A clear separation between signal and noise can be observed. During operation, the PMT gain calibration is monitored by a

\footnotetext{
${ }^{*}$ Corresponding author

Email address: flasagni@cern.ch (F. Lasagni Manghi)
}

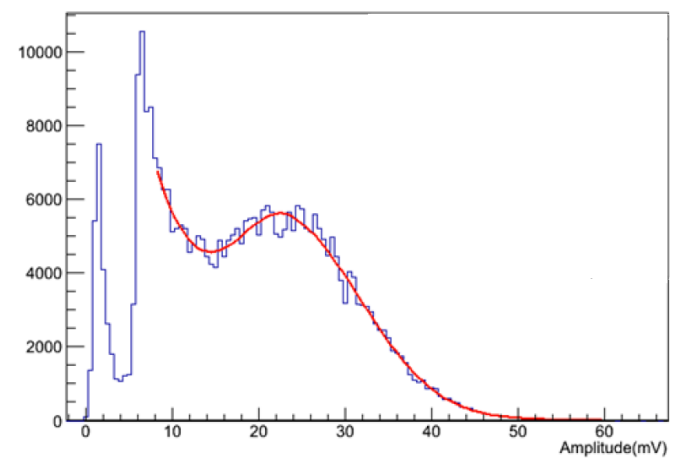

Figure 1: Amplitude spectrum from a bismuth source using a R760 Hamamatsu PMT, with the baseline clearly visible to the left.

redundant system made of:

- optical fibers carrying LED signals;

- optical fibers carrying LASER signals provided by the calibration system of the Tile calorimeter;

- radioactive sources $(\mathrm{Bi}-207)$.

\section{Electronics}

The old LUCID electronics could not fully cope with the 25 ns bunch-spacing since the electronic front-end system was far from the detector, resulting in pulses with tails beyond the 25 ns. Early signal digitization performed by new front-end electronics placed next to the PMTs will avoid this effect, without need for amplification and shaping. The charge integration method performed by the new electronics was proven to be free from the main systematics affecting the old system and enables an innovative algorithm based on the total charge collected by the PMTs. New VME boards (LUCROD), placed 
close to the detector, perform early signal digitization as well as PMT-charge integration over each bunch crossing time (25 ns). Each board is provided with 16 input channels, each connected to a different PMT. The main components of the LUCROD board are shown in Fig. 2, together with a schema of the complete system, where each channel is connected to a low noise amplifier and a $480 \mathrm{MHz}$ FADC which is used both for charge measurement and as a discriminator for "hit" definition. The FPGAs integrate the PMT signals over each bunch crossing, perform a first series of luminosity algorithms, including charge integration, and sends the pattern of over-threshold PMTs, the "hits" to the LUMAT board via optical link communication. Samples of the input signals can be collected for monitoring purposes. The LUMAT boards are entitled with the task

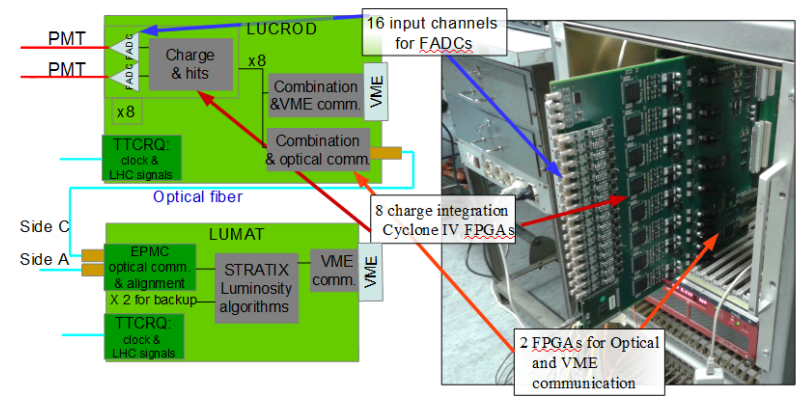

Figure 2: The LUCID electronics system with the LUCROD board.

of combining data from the two detectors to produce on-line and off-line luminosity measurements. Twelve different algorithms are performed using the "hit" patterns received from the LUCROD boards. This structure of the electronic system ensures maximum flexibility and reliability, thanks to redundancy and the use of both new, more accurate, methods and proven, reliable ones.

To further enhance redundancy and stability, part of the system sends signals to the old LUCID system for it to work in parallel and cross-check the first Run 2 results.

\section{First collisions at LHC}

The LHC was turned on after the shut-down period in April and LUCID successfully registered the first events, showing its ability to measure luminosity on a bunch-by-bunch basis (each bunch measurement is still integrated over a an interval of $60 s$ ), as portrayed in Fig. 3.

The first beam collisions, in May, are shown in Fig. 4 using the LUMAT Event-OR algorithm, that counts any event registering a hit in a tube in any detector.

\section{Future Developments and Conclusions}

An implementation of digital filtering in the LUCROD FPGA is under study in order to improve the signal reconstruction for optimal charge measurement. The algorithms for digi-

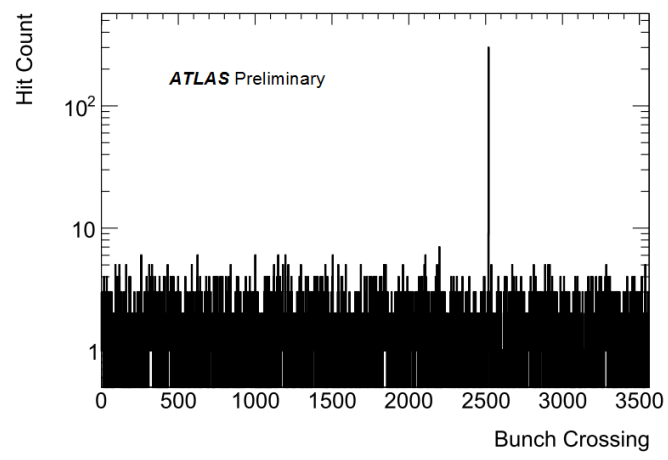

Figure 3: LUCROD PMT-1 hit counts as a function of bunch crossing number in so-called beam splash events. The constant background is given by the calibration Bi-207 source.[4]

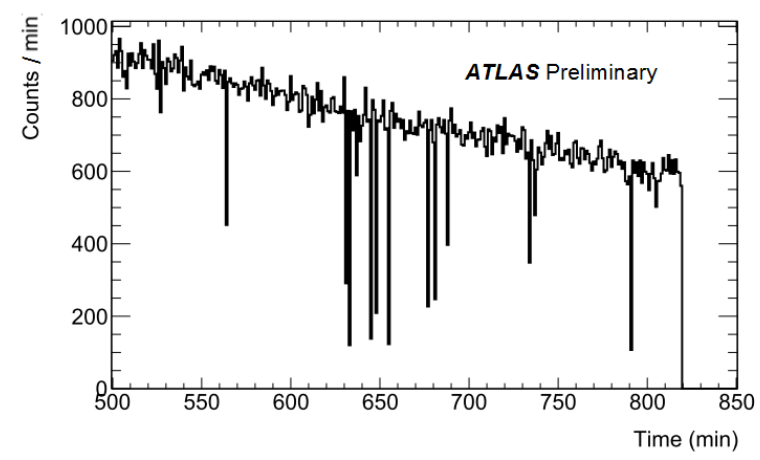

Figure 4: The first luminosity measurement of collision data at $\sqrt{s}=900 \mathrm{GeV}$, as a function of time. [4]

tal filtering will be optimized in an off-line analysis of the LUCROD signal shapes. This technique can also be used in other fields to improve detector resolution, for example the energy resolution of X-Ray detectors.

The main features of the LUCID detector are fully functional and have already proven to be working correctly. New features and improvements are under development to provide better linearity of the luminosity measurements and additional information to the ATLAS detector.

\section{References}

[1] ATLAS Collaboration. The ATLAS Experiment at the CERN Large Hadron Collider. JINST, 3:S08003, 2008.

[2] ATLAS Collaboration. Improved luminosity determination in pp collisions at $\operatorname{sqrt}(\mathrm{s})=7 \mathrm{TeV}$ using the ATLAS detector at the LHC. Eur.Phys.J., C73(8):2518, 2013.

[3] Federico Lasagni Manghi. LUCID upgrade - ATLAS luminosity monitor for the 2015 LHC. PoS, TIPP2014:317, 2014.

[4] ATLAS Collaboration. Public forward detector plots for collision data. https://twiki.cern.ch/twiki/bin/view/AtlasPublic/ ForwardDetPublicResults\#LUCID_figures. 